\title{
Analytical Synthesis of Fully Canonical Cascaded-Doublet Prototype Filters
}

\author{
Steven Caicedo Mejillones, Matteo Oldoni, Stefano Moscato, and Giuseppe Macchiarella, Fellow, IEEE
}

\begin{abstract}
This letter presents an analytical procedure for the synthesis of a low-pass prototype filter constituted by the cascade of doublet blocks coupled by inverters or shunt reactances. The considered doublet is a second order resonating structure composed of two resonant and two non-resonant nodes which allows the placement of two finite transmission zeros in the frequency response. The considered configuration allows realizing compact fully canonical filters with the maximum number of transmission zeros (the doublets are typically implemented through dual-mode cavities). The proposed procedure introduces for the first time an analytical procedure for synthesizing the low-pass prototype of the cascaded-block topology, overcoming the time consuming and inaccurate procedures currently based on numerical optimization. Two synthesis examples are presented to validate the novel synthesis procedure.
\end{abstract} sis.

Index Terms-Elliptic filters, doublets, cascaded-block synthe-

\section{INTRODUCTION}

$\mathbf{T}$ HE design of highly selective filters may require the introduction in the response of several transmission zeros (TZs), which makes their synthesis complex and their implementation generally difficult and cumbersome. A popular solution, which makes practical the adjustment of the designed filter, is the cascaded-block configuration using cross-coupled resonator blocks [1]. Such a topology, whose low-pass prototype can be synthesized with the methods available in the literature [1-4], has however a relevant limitation: the number of transmission zeros cannot be equal to the filter order (i.e. a fully canonical response cannot be realized). To overcome this limitation, solutions have appeared in the literature where the cascaded configuration includes both resonating $(\mathrm{RN})$ and nonresonating (NRN) nodes [5-12]. In this way each block can be fully canonical, as well as the whole cascaded configuration. Examples of fully canonical blocks are represented by the socalled singlets and doublets, generally implemented in waveguide technology [8-11]. Recently, a procedure for the synthesis of cascaded-singlet fully canonical filters has been presented in [12], but no procedure is till now available for cascadeddoublets. In fact, the design of such filters in the literature is

Manuscript received March 09, 2020; revised April 19 and June 27, 2020; accepted July 22, 2020. Date of publication October 1, 2020. This work is funded by the H2020 research program 5G STEP-FWD under grant agreement No. 722429.

S. Caicedo, M. Oldoni and S. Moscato are with R\&D Dept., SIAE MICROELETTRONICA, 20093 Cologno Monzese (MI), Italy (e-mail: [steven.caicedo, matteo.oldoni, stefano.moscato]@siaemic.com). S. Caicedo and G. Macchiarella are with Dipartimento di Elettronica, Informazione e Bioingegneria, POLITECNICO DI MILANO, 20133 Milano (MI), Italy (email: [stevenkleber.caicedo, giuseppe.macchiarella]@polimi.it).

Color versions of one or more of the figures in this letter are available online at http://ieeexplore.iee.org.

Accepted version. Digital Object Identifier 10.1109/LMWC.2020.3026187. based on the evaluation of the coupling matrix by means of optimization techniques [9]. To overcome this approach (which implies no certainty in the final result, limited accuracy and longer computation time), we present in this work an analytical solution for the synthesis of a cascaded-doublet prototype filter. The considered doublet is schematically represented in Fig. 1, with reference to the low-pass model of the filter.

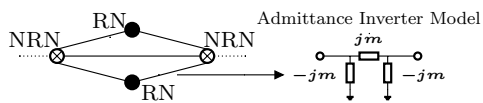

Fig. 1. Routing scheme of the doublet (low-pass domain). Black circles represent unit capacitance in parallel to frequency-independent susceptances (resonating nodes RN). White crossed circles: frequency-independent susceptances (non-resonating nodes NRN). Black lines: admittance inverters.

\section{SyNTHESIS METHOD}

The proposed procedure starts with the synthesis of a fully canonical extracted-pole prototype of even order, using one of the methods available in the literature [13]. Fig. 2 shows the routing scheme of the synthesized low-pass prototype of order $n$, which is partitioned into second-order cascaded blocks.

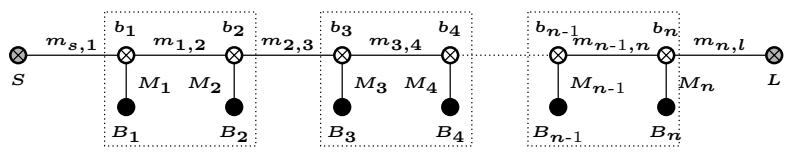

Fig. 2. Routing scheme of an extracted-pole low-pass prototype, partitioned into second-order blocks (dotted rectangles). Source $S$ and load $L$ are assumed unit conductance. White crossed nodes are NRNs: shunt susceptances $\left(b_{i}\right)$. Black nodes are RNs: susceptances $\left(B_{i}\right)$ in parallel to a unit capacitance.

Let consider the inverters $m_{k, k+1}$ ( $k$ even) separating the dotted rectangles in Fig. 2. Two solutions can be devised for replacing these inverters with an equivalent representation (Fig. 3). Note that in the first solution (Fig. 3a), the two susceptances $b_{k, k+1}^{\mathrm{A}}$ and $b_{k, k+1}^{\mathrm{C}}$ must be embedded into the NRNs adjacent to the inverter $m_{k, k+1}$.

(a)

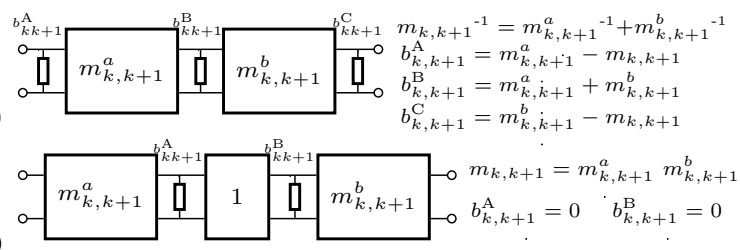

Fig. 3. Equivalent representation for the inverters $m_{k, k+1}$ (k even) separating the extracted-pole blocks in Fig. 2. Split into (a) 2 inverters, (b) 3 inverters. 
Both solutions in Fig. 3 imply that either inverter $\left(m_{k, k+1}^{a}\right.$ or $m_{k, k+1}^{b}$ ) can be arbitrarily assigned as the other parameters are determined through the relationships shown in Fig. 3. This also means that the final synthesized network is not unique.

The first and last inverters $\left(m_{s, 1}, m_{n, l}\right)$ are instead replaced with the cascade of two inverters (they behave as transformers). Assigning the first (last) unitary $\left(m_{s, 1}^{a}=m_{n, l}^{b}=1\right)$ :

$$
m_{s, 1}^{b}=m_{s, 1}, \quad m_{n, l}^{a}=m_{n, l} .
$$

Fig. 4 shows the modified extracted-pole network after the replacement of the inverters $m_{k, k+1}$. Note that the partitioned blocks in Fig. 4 include $m_{k, k+1}^{a}$ and $m_{k, k+1}^{b}$ inverters, and the blocks are separated by either NRNs (first case, Fig. 4a) or by a unit inverter (second case, Fig. 4b).

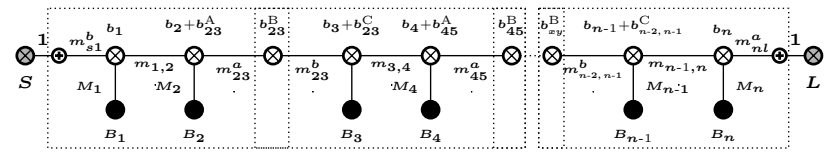

(a)

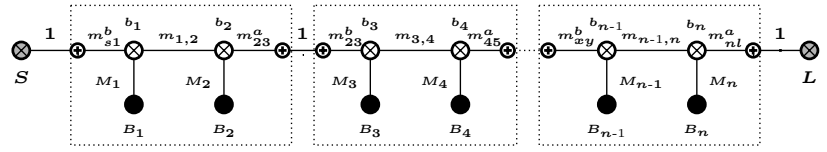

(b)

Fig. 4. Routing scheme of an extracted-pole low-pass prototype after splitting the inverters that connect the second order blocks according to the two corresponding cases in Fig 3, to synthesize a filter with adjacent doublets that (a) share an NRN, (b) are coupled by means of a unit inverter. White circles with (+) represent zero NRNs.

The next goal is to transform each partitioned block into the doublet topology shown in Fig. 1. To simplify the representation, the generic partitioned block is assumed with the parameters reported in Fig 5a.

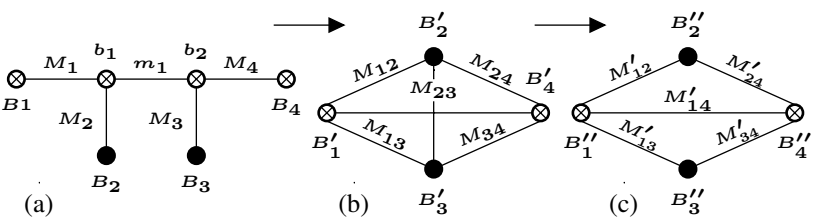

Fig. 5. Transformation from (a) extracted-pole to (c) doublet. (b) After starto-mesh conversion. Relations are in (2)-(4), limited to 2-order blocks.

The first transformation (Fig. 5b), exploits a double star-tomesh conversion [14-15] applied in sequence to nodes $b_{1}$ and $b_{2}$. The following closed form expressions have been derived for the elements of the second order transformed network:

$$
\begin{aligned}
& \left(\begin{array}{cccc}
B_{1}^{\prime} & M_{12} & M_{13} & M_{14} \\
M_{12} & B_{2}^{\prime} & M_{23} & M_{24} \\
M_{13} & M_{23} & B_{3}^{\prime} & M_{34} \\
M_{14} & M_{24} & M_{34} & B_{4}^{\prime}
\end{array}\right)=\left(\begin{array}{ccccc}
B_{1} & 0 & 0 & 0 \\
0 & B_{2} & 0 & 0 \\
0 & 0 & B_{3} & 0 \\
0 & 0 & 0 & B_{4}
\end{array}\right)+ \\
& \left(\begin{array}{ccccc}
-b_{2} M_{1}^{2} & -b_{2} M_{1} M_{2} & M_{1} M_{3} m_{1} & M_{1} M_{4} m_{1} \\
-b_{2} M_{1} M_{2} & -b_{2} M_{2}^{2} & M_{2} M_{3} m_{1} & M_{2} M_{4} m_{1} \\
M_{1} M_{3} m_{1} & M_{2} M_{3} m_{1} & -b_{1} M_{3}^{2} & -b_{1} M_{3} M_{4} \\
M_{1} M_{4} m_{1} & M_{2} M_{4} m_{1} & -b_{1} M_{3} M_{4} & -b_{1} M_{4}^{2}
\end{array}\right)
\end{aligned}
$$

The topology in Fig. 5b can be transformed into a doublet (Fig. 5c) by annihilating the coupling $M_{23}$ by means of a matrix rotation [16] with pivot $(2,3)$, which does not affect the capacitances of the two RNs. Annihilation of $M_{23}$ requires the following rotation angle:

$$
\theta=\frac{1}{2} \tan ^{-1}\left(\frac{2 M_{23}}{B_{3}^{\prime}-B_{2}^{\prime}}\right)
$$

After rotation, we finally obtain the elements of the block that defines the doublet topology (Fig. 5c):

$$
\begin{array}{r}
B_{1}^{\prime \prime}=B_{1}^{\prime}, B_{2}^{\prime \prime}=c\left(B_{2}^{\prime} c-M_{23} s\right)-s\left(M_{23} c-B_{3}^{\prime} s\right), \\
B_{4}^{\prime \prime}=B_{4}^{\prime}, B_{3}^{\prime \prime}=c\left(B_{3}^{\prime} c+M_{23} s\right)+s\left(M_{23} c+B_{2}^{\prime} s\right), \\
M_{12}^{\prime}=M_{12} c-M_{13} s, M_{13}^{\prime}=M_{13} c+M_{12} s, \\
M_{24}^{\prime}=M_{24} c-M_{34} s, M_{34}^{\prime}=M_{34} c+M_{24} s, \\
M_{23}^{\prime}=0, M_{14}^{\prime}=M_{14} \text {, with } c=\cos (\theta), s=\sin (\theta) .
\end{array}
$$

Once all the partitioned blocks in Fig. 4 have been transformed, the final cascaded-doublet topology can be represented as in Fig. 6 (both cases). Note that these transformations do not affect the capacitances of the RNs, which remain unitary.

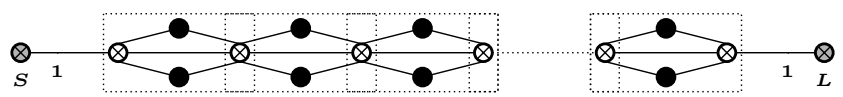

(a)

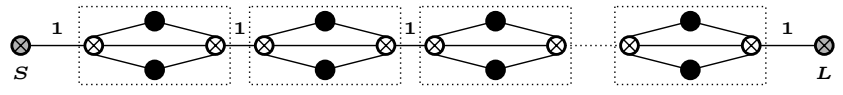

(b)

Fig. 6. Cascaded-doublet topology. (a) Adjacent doublets share an NRN. (b) Each doublet is coupled with the adjacent ones by means of a unit inverter.

\section{UNIVERSAL COEFFICIENTS}

It is known [1] that the coupling matrix $M$ characterizing a prototype filter with arbitrary topology is invariant to scaling rows and columns for an arbitrary constant (the nodes capacitance must be multiplied by the same constant squared). Exploiting this property, we introduce the following universal coefficients that are invariant to the scaling operation:

$$
\begin{aligned}
k_{i, j} & =\frac{M_{i, j}}{\sqrt{\left|M_{i, i} M_{j, j}\right|}}(i \neq S, j \neq L), \quad \Omega_{i}=\frac{M_{i, i}}{C_{i}}(R N) \\
k_{T, j} & =\frac{M_{T, j}}{\sqrt{\left|M_{j, j}\right|}}(T=S, L), \quad S_{i}=\operatorname{Sign}\left(M_{i, i}\right)(N R N),
\end{aligned}
$$

where $C_{i}$ is the capacitance of $i$-th node $\left(C_{i}=0\right.$ for NRN). $M_{i, j}$ are the elements of the matrix $M$. The synthesis procedure of cascaded doublets proposed here requires assigning arbitrary parameters, which affect the coupling matrix of the synthesized prototype. However, although infinite different solutions can be found for this matrix, we may wonder about the unicity of the synthesis result. This property can be referred, for instance, to the scaling concept introduced above. In fact, we have found that the first topology here considered (Fig. 6a) is not unique while the second topology (Fig. 6b) is unique, 
i.e. the computed universal coefficients do not depend on the initially assigned inverters parameters $\left(m_{k, k+1}^{a}\right.$ or $\left.m_{k, k+1}^{b}\right)$. In any case, once the synthesis of one of the cascaded-coupled topologies has been carried out (with arbitrary assignment of the free parameters), the universal coefficients allow exploiting the available degrees of freedom by assigning freely some of the filter elements and computing consequently the others.

\section{VERIFICATIONS}

\section{A. Filter with 2 poles and 2 Transmission Zeros}

Consider a 2 poles Chebyshev filter with central frequency $(F c)$ at $10 \mathrm{GHz}$, fractional bandwidth (FB) of $1.5 \%, 20 \mathrm{~dB}$ of return loss (RL) and transmission zeros (TZs) at [9.636, $9.796] \mathrm{GHz}$. No free parameter must be assigned in this case to perform the doublet synthesis. The results of the three steps of the novel procedure are reported in Fig. 7.
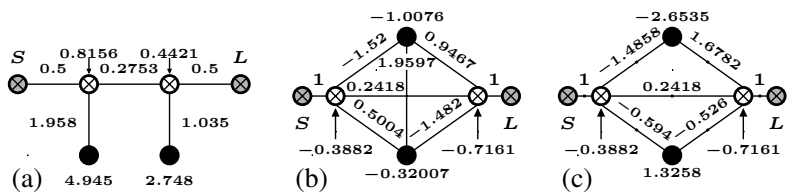

Fig. 7. Synthesized doublet (coupling matrices). (a) Extracted-pole block. (b) After Star-to-Mesh transformations. (c) After $M_{23}$ annihilation (doublet).

The scattering parameters of the final doublet are reported in Fig. 8, together with the evaluated universal coefficients. It was implemented with a TM dual-mode cavity (dimensions and simulations are in Fig. 8a), where the resonant modes (RM) $\mathrm{TM}_{120}, \mathrm{TM}_{210}$ are the RNs; the slots are the NRNs; and the non-RM TE $10, \mathrm{TE}_{01}, \mathrm{TM}_{11}$ are the couplings [9] [17].

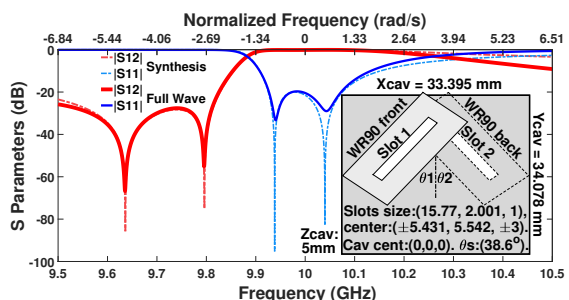

(a)

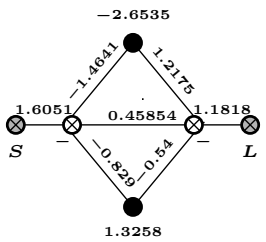

(b)
Fig. 8. (a) Computed scattering parameters. (b) Universal coefficients of the doublet (NRNs are characterized by $\operatorname{Sign}\left(M_{i, i}\right)$, RNs by the ratio $M_{i, i} / C_{i}$ ).

\section{B. Filter with 6 poles and 6 Transmission Zeros}

The specifications considered here are the same used in [9] for the physical dimensioning of a three-cavity TM dual-mode filter discussed in that work: $F c$ at $10 \mathrm{GHz}, \mathrm{FB}$ of $1.5 \%$, RL of $20 \mathrm{~dB}$, TZs at $[9.653,9.877,10.15,10.39,9.697,9.84] \mathrm{GHz}$. The synthesis of both considered cascaded-doublet topologies has been performed and the computed universal coefficients are reported in Fig. 9, together with the filter response (including full-wave simulations of the three-cavity dual-mode filter connected through slots [9]). For both cases, the order given by the TZs specifications is the same as in the circuits ( 2 TZs per doublet/cavity). From Fig. 9a we observe that the response of the synthesis is perfectly equi-ripple in the passband, in contrast to the one presented in [9], where the evaluation of the coupling matrix by optimization has not allowed this result (although RL $>20 \mathrm{~dB}$ in the passband).

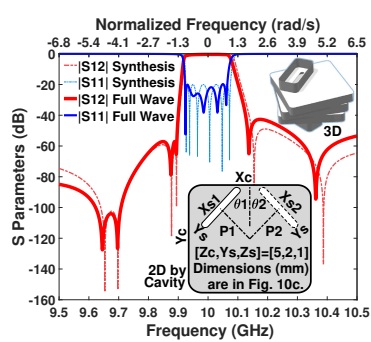

(a)

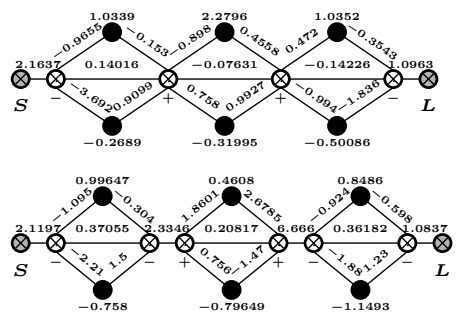

(b)
Fig. 9. (a) Computed response of the cascaded-doublet filter. (b) Synthesized routing schemes (universal coefficients, the sign of the NRNs is also reported).

Another point to remark is that, thanks to the universal coefficients here introduced, we can obtain a final prototype that respects the constraints imposed by the actual physical structure. For instance, the second topology can be obtained with all the inverters connecting the doublets assigned to one (equivalent to quarter-wavelength waveguide sections [9]). The first topology can be instead obtained with all NRNs set to 1 . The coupling matrix elements for both cases are reported in Fig. 10, while the filter response is still Fig. 9a. Finally, it is noteworthy that the synthesized cascaded doublets can be also implemented in SIW [10] and microstrip [18] technologies.

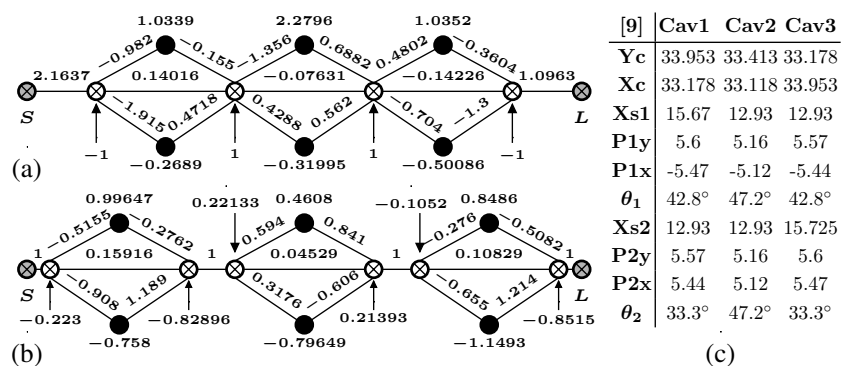

Fig. 10. Coupling matrix elements from the universal coefficients. (a) Topology with all NRNs magnitude set to 1 . (b) Topology with inverters connecting the doublets set to 1 . (c) Dimensions (mm) of the filter (Fig. 9a).

\section{CONCLUSIONS}

In this letter, a novel synthesis procedure for cascadeddoublet prototype filters has been introduced. To the Authors' knowledge, this is the first analytical solution for the considered topology, whose coupling matrix could previously only be obtained by optimization. The synthesis of two cascaded configurations has been developed, the first using NRNs for separating the doublets, the latter using inverters. In both cases, the novel synthesis procedure requires the assignment of arbitrary parameters. For the second cascaded topology introduced, we have shown that all the possible solutions depending on the initial free parameters are equivalent as the computed coupling matrices are related by a scaling operation. We have validated the novel synthesis procedure by synthesizing a 6-order fully canonical filter previously designed by optimization. 


\section{REFERENCES}

[1] R. J. Cameron, C. M. Kudsia, and R. R. Mansour, Microwave Filters for Communication Systems. Hoboken, NJ, USA: John Wiley \& Sons, 2007

[2] T. Reeves, N. Van Stigt and C. Rossiter, "A method for the direct synthesis of general sections," 2001 IEEE MTT-S International Microwave Sympsoium Digest , Phoenix, AZ, 2001, pp. 1471-1474 vol.3.

[3] N. Yildirim, O. A. Sen, Y. Sen, M. Karaaslan, and D. Pelz, "A revision of cascade synthesis theory covering cross-coupled filters," IEEE Trans. Microw. Theory Techn., vol. 50, no. 6, pp. 1536-1543, June 2002.

[4] S. Tamiazzo and G. Macchiarella, "An analytical technique for the synthesis of cascaded N-tuplets cross-coupled resonators microwave filters using matrix rotations," IEEE Trans. Microw. Theory Techn., vol.53, no. 5, pp. 1693-1698, May 2005.

[5] S. Amari, U. Rosenberg, and J. Bornemann, "Singlets, cascaded singlets and the nonresonating node model for modular design of advanced microwave filters," IEEE Microw. Wireless Compon. Lett., vol. 14, no. 5, pp. 237-239, May 2004.

[6] R. Wu, S. Amari and U. Rosenberg "Cross-Coupled Microstrip Band Reject Filters With Non-Resonating Nodes," IEEE Microw. Wireless Compon. Lett., vol. 15, no. 9, pp. 585-587, Sept. 2005.

[7] A. Garcia-Lamperez, S. Llorente-Romano and M. Salazar-Palma, "Analytical Coupled-Resonator Filter Synthesis Method by Extraction of Fully Canonical Second Order Blocks," IEEE Microw. Wireless Compon. Lett., vol. 23, no. 3, pp. 137-139, Mar. 2013

[8] V. Nocella, L. Pelliccia, C. Tomassoni, R. Sorrentino, "Miniaturized Dual-Band Waveguide Filter Using TM Dielectric-Loaded Dual-Mode Cavities," IEEE Microw. Wireless Compon. Lett., vol. 26, no. 5, pp. 310-312, May 2016.
[9] C. Tomassoni, S. Bastioli, R. Sorrentino, "Generalized TM Dual-Mode Cavity Filters" IEEE Trans. Microw. Theory Techn., vol.59, no. 12, pp. 3338-3346, Dec. 2011

[10] S. Sirci, M. Sanchez-Soriano, J. Martinez, V. Boria "Advanced Filtering Solutions in Coaxial SIW Technology Based on Singlets, Cascaded Singlets, and Doublets," IEEE Access, vol. 7, Mar. 2019.

[11] M. Esmaeili and J. Bornemann, "Substrate Integrated Waveguide TriplePassband Dual-Stopband Filter Using Six Cascaded Singlets," IEEE Microw. Wireless Compon. Lett., vol. 24, no. 7, pp. 439-441, July 2014.

[12] G. Macchiarella, G. G. Gentili, C. Tomassoni, S. Bastioli and R. V. Snyder, "Design of Waveguide Filters With Cascaded Singlets Through a Synthesis-Based Approach," IEEE Trans. Microw. Theory Techn., vol. 68, no. 6, pp. 2308-2319, June 2020.

[13] S. Amari, G. Macchiarella, "Synthesis of In-Line Filters With Arbitrarily Placed Attenuation Poles by Using Non-Resonating Nodes," IEEE Trans. Microw. Theory Tech., vol. 52, n.10, pp. 3075-3081, Oct. 2005.

[14] M. van Lier and R. Otten, "Planarization by transformation," IEEE Trans. Circuit Theory, vol. 20, no. 2, pp. 169-171, March 1973.

[15] S. Bedrosian, "Converse of the Star-Mesh Transformation," IEEE Trans. Circuit Theory, vol. 8, no. 4, pp. 491-493, December 1961.

[16] R. J. Cameron, "General coupling matrix synthesis methods for Chebyshev filtering functions," in IEEE Trans. Microw. Theory Techn., vol. 47, no. 4, pp. 433-442, April 1999.

[17] S. Bastioli, "Nonresonating Mode Waveguide Filters," IEEE Microwave Magazine, vol. 12, no. 6, pp. 77-86, Oct. 2011.

[18] W. Tang and J. Hong, "Quasi-elliptic function doublet filters without cross coupling," 2009 European Microwave Conference (EuMC), Rome, 2009, pp. 452-455. 